\title{
Predicting and controlling the Ebola infection
}

\author{
Amira Rachah ${ }^{\dagger}$ and Delfim F. M. Torres ${ }^{\ddagger} *$
}

\begin{abstract}
We present a comparison between two different mathematical models used in the description of the Ebola virus propagation currently occurring in West Africa. In order to improve the prediction and the control of the propagation of the virus, numerical simulations and optimal control of the two models for Ebola are investigated. In particular, we study when the two models generate similar results.
\end{abstract}

Keywords: Mathematical modelling; Epidemiology; Ebola; Optimal control analysis.

\section{Introduction}

Ebola virus is a severe infection affecting currently several African countries, mainly Guinea, Sierra Leone, and Liberia. It was first discovered in 1976 in the Democratic Republic of Congo, near the Ebola river, where the virus takes its name, but recently was also identified in West Africa [1,2]. The latest outbreak of the virus was not only the largest, it is also the deadliest infectious disease outbreak ever known. According to the World Health Organisation (WHO), "it is thought that fruit bats of the Pteropodidae family are natural Ebola virus hosts". It then spreads through human-to-human transmission, becoming the deadliest pathogen. The early signs and symptoms of the virus include a sudden onset of fever, intense weakness and headache. Over time, symptoms become increasingly severe and include diarrhea, raised rash, internal and external bleeding, from nose, mouth, eyes and anus. As the virus spreads through the body, it damages the immune system and organs [3-7]. Ebola virus is transmitted to an initial human by contact with an infected animal's body fluid. On the other hand, human-to-human transmission can take place with direct contact (through broken skin or mucous membranes in, for example, the eyes, nose, or mouth) with blood or body fluids of a person who is sick with or has died from Ebola. It is also transmitted indirectly via exposure to objects or environment contaminated with infected secretions [8-13]. There is yet no licensed treatment proven to neutralise the virus, but a range of blood, immunological and drug therapies are under development.

Mathematical models are a powerful tool for investigating human infectious diseases, such as Ebola virus, contributing to the understanding of the dynamics of disease and providing useful predictions about the potential transmission of a disease and the effectiveness of possible control measures, which can provide valuable information for public health policy makers [14-16]. Cases of success include influenza and small pox, for which epidemic models have provided the foundation for the best vaccination practices $[17,18]$. Epidemic models date back to the early twentieth century, to a set of three articles from 1927, 1932, and 1933 by Kermack and McKendrick, whose models were used for modelling the plague and cholera epidemics [19-21]. The most commonly implemented models in epidemiology are the SIR and SEIR models. The SIR model consists of three compartments: Susceptible individuals $S$, Infectious individuals I, and Recovered individuals $R$. In many infectious diseases there is an exposed period after the transmission of the infection from susceptible to potentially infective members but before these potential infective can transmit infection. Then an extra compartment is introduced, the so called exposed class $E$, and we use compartments $S$, $E, I$ and $R$ to give a generalization of the basic SIR model $[22,23]$.

When analyzing a new outbreak, the researchers usually start with the SIR and SEIR models to fit the available outbreak data, obtaining estimates for the parameters of the model [23]. In the modelling of the spreading mechanism of the Ebola virus currently affecting several African countries, Rachah and Torres used the SIR [24] and the SEIR [25] basic models. In their work, they used parameters identified from recent data of the WHO to describe the behavior of the virus. After modelling and simulation steps, they applied optimal control techniques in order to understand how the spread of the virus may be controlled, e.g., through education campaigns, immunization or isolation [26-31].

Here we discuss the differences between the SIR and SEIR models, previously presented by Rachah and Torres in their study of the description of the behavior of the Ebola virus [24, 25]. For a non-integer order (fractional) SEIR model see [32]. Our

\footnotetext{
Université Paul Sabatier, Institut de Mathématiques, 31062 Toulouse Cedex 9, France. ${ }^{\ddagger}$ Center for Research and Development in Mathematics and Applications (CIDMA), Department of Mathematics, University of Aveiro, 3810-193 Aveiro, Portugal.

* Correspondence to: Department of Mathematics, University of Aveiro, 3810-193 Aveiro, Portugal. Email: delfim@ua.pt
} 
comparison is based on the numerical resolution of the models. In order to study which model can better control the propagation of the virus into population, we discuss the comparison between the SIR and SEIR models by studying the optimal control of the Ebola virus. The manuscript is organized as follows. In Section 2 we briefly recall the SIR and SEIR models for Ebola infection. Their numerical simulation is carried out in Section 3, where we emphasise the differences between them. The best strategies for the control of the propagation of Ebola into the populations, accordingly to both models, are investigated in Section 4 . We end with Section 5 of conclusion.

\section{The SIR and SEIR models for Ebola infection}

SIR and SEIR models provide the foundations of mathematical modelling in epidemiology, being widely used in practice, to describe real world problems in epidemiology. In this section, we briefly describe the two mathematical models.

In the modelling of Ebola virus, Rachah and Torres [24] used the basic SIR (Susceptible-Infectious-Recovery) model. In the description of the transmission of Ebola virus by the SIR model, the population is divided into three groups:

- the Susceptible compartment $S(t)$ denotes individuals who are susceptible to catch the virus, and so might become infectious if exposed;

- the Infectious compartment $I(t)$ denotes infectious individuals who are suffering the symptoms of Ebola and are able to spread the virus through contact with the susceptible class of individuals;

- the "Recovered" compartment $R(t)$ denotes individuals that do not affect the transmission dynamics in any way.

The model is described by the following system of nonlinear ordinary differential equations (ODEs):

$$
\left\{\begin{array}{l}
\frac{d S(t)}{d t}=-\beta S(t) I(t), \\
\frac{d I(t)}{d t}=\beta S(t) I(t)-\mu I(t), \\
\frac{d R(t)}{d t}=\mu I(t),
\end{array}\right.
$$

where $\beta>0$ is the infection rate and $\mu>0$ is the recovered rate. The initial conditions are given by

$$
S(0)=S_{0}>0, \quad I(0)=I_{0}>0, \quad R(0)=0
$$

The SEIR model is an extension of the basic SIR model, where an extra compartment is introduced, the so called exposed compartment $\hat{E}(t)$. The Exposed compartment $\hat{E}(t)$ denotes the individuals who are infected but the symptoms of the virus are not yet visible. The transmission of the virus is then described by the following set of nonlinear ODEs:

$$
\left\{\begin{array}{l}
\frac{d \hat{S}(t)}{d t}=-\hat{\beta} \hat{S}(t) \hat{I}(t) \\
\frac{d \hat{E}(t)}{d t}=\hat{\beta} \hat{S}(t) \hat{I}(t)-\hat{\gamma} \hat{E}(t), \\
\frac{d \hat{I}(t)}{d t}=\hat{\gamma} \hat{E}(t)-\hat{\mu} \hat{I}(t), \\
\frac{d \hat{R}(t)}{d t}=\hat{\mu} \hat{I}(t)
\end{array}\right.
$$

where $\hat{\beta}>0$ is the contact rate (transmission rate), $\hat{\gamma}$ is the infection rate, and $\hat{\mu}>0$ is the recovered rate. The initial conditions are given by

$$
\hat{S}(0)=\hat{S}_{0}>0, \quad \hat{E}(0)=\hat{E}_{0} \geq 0, \quad \hat{I}(0)=\hat{l}_{0}>0, \quad \hat{R}(0)=0 .
$$

The particularity of the SEIR model is in the exposed compartment, which is characterized by infected individuals that cannot communicate the virus. These individuals are in the so called latent period [23]. For Ebola virus, such stage makes all sense since it takes a certain time for an infected individual to become infectious. During this period of time such individuals are in the exposed compartment $\hat{E}(t)$, before they become infectious. The infectious compartment at time $t$, denoted by $\hat{\imath}(t)$, represents the individuals that are infected by the virus and are able to spread it through contact with the susceptible class of individuals. Finally, we have the "recovered" compartment $\hat{R}(t)$, which denotes the individuals that do not affect the transmission dynamics in any way. Figure 1 shows the diagrammatic representation of the virus progress in an individual.

\subsection{Analysis of the SIR model}

In this subsection we study the properties of the SIR model (1)-(2). We begin with a trivial observation. 


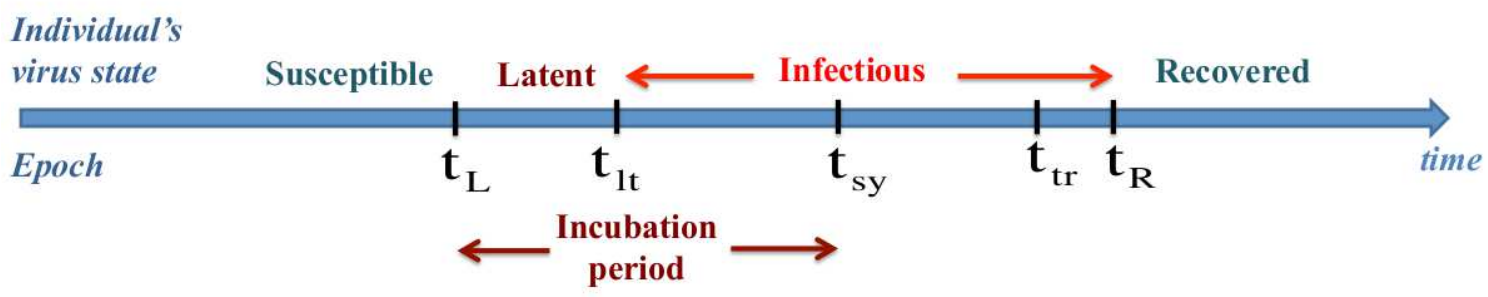

Figure 1. Ebola virus progress in an individual by using the SEIR model, where $t_{L}$ is the time when infectious occurs, $t_{l t}$ denotes latency to infectious transition, $t_{s y}$ the time when symptoms appear, $t_{t r}$ the time of first transmission to another susceptible, and $t_{R}$ the time where the individual is no longer infectious.

Proposition 2.1 The total population $N$ being modelled by the SIR model (1) is constant in time, that is, $S(t)+I(t)+R(t)=N$ for all $t \geq 0$.

Proof Follows by adding the right-hand side of equations $(1): S^{\prime}(t)+I^{\prime}(t)+R^{\prime}(t)=0$.

The following lemmas provide essential properties of the SIR model (1)-(2).

Lemma 2.1 Let $(S(t), I(t), R(t))$ be a solution of the SIR model $(1)-(2)$. Then $S(t) \geq 0$ and $I(t) \geq 0$ for all $t \geq 0$.

Proof The proof is done by contradiction. We have that $S(t)$ and $I(t)$ are continuous functions, $S(0)=S_{0}>0$ and $I(0)=I_{0}>0$. Suppose that $S\left(t^{\prime}\right)<0$. Then, by the intermediate value theorem, there exist $t_{1} \in\left[0, t^{\prime}\right]$ such that $S\left(t_{1}\right)=0$. By using the first equation of $(1)$, we obtain that $S(t)=S\left(t_{1}\right) e^{f}=0$ for $t \geq t_{1}$, where $f$ is the primitive of $-\beta /(t)$. Thus $S(t)=0$ for $t \geq t_{1}$, which is a contradiction. The same arguments can be applied to $I(t)$.

Lemma 2.2 Let $(S(t), I(t), R(t))$ be a solution of the SIR model (1)-(2). Then $S(t)+I(t) \leq N$ and $0 \leq R(t) \leq N$ for all $t \geq 0$.

Proof The sum of the first two equations of (1) give us $\frac{d}{d t}[S(t)+I(t)]=-\mu I(t) \leq 0$. Now, by using Lemma 2.1 and $S(0)+I(0)=N$, we obtain that $S(t)+I(t) \leq N$. From Proposition 2.1 we have $N-S(t)-I(t)=R(t)$. We conclude that $R(t) \geq 0$. From Lemma 2.1, we have $S(t)+I(t) \geq 0$, which implies that $N-S(t)-I(t) \leq N$. Therefore, $R(t)=$ $N-S(t)-I(t) \leq N$.

In what follows we use $p$ to denote the relative removal rate parameter.

Definition 2.1 Given a SIR model (1), we introduce the quantity $p:=\frac{\mu}{\beta}$, called the relative removal rate parameter.

Lemma 2.3 Let $(S(t), I(t), R(t))$ be a solution of the SIR model $(1)-(2)$. Then, $I(S(t))=I_{0}+S_{0}-S(t)+p \ln \left(\frac{S(t)}{S_{0}}\right)$.

Proof From (1)-(2) one has

$$
\frac{d l}{d S}=\frac{\beta S(t)-\mu}{-\beta S(t)}=\frac{\mu}{\beta S(t)}-1
$$

By integrating $d I=\left[\frac{\mu}{\beta S(t)}-1\right] d S$, we obtain that

$$
\int_{0}^{t} d I=\int_{0}^{t}\left[\frac{\mu}{\beta S(t)}-1\right] d S
$$

so that $I(t)-I_{0}=\frac{\mu}{\beta} \ln (S(t))-\frac{\mu}{\beta} \ln \left(S_{0}\right)-S(t)-S_{0}$. Therefore, $I(S(t))=I_{0}+S_{0}-S(t)+p \ln \left(\frac{S(t)}{S_{0}}\right)$, where $p=\frac{\mu}{\beta}$.

From Lemma 2.3, we know that the quantity $\frac{\mu}{\beta} S-1=p S-1$ is positive if $S<p$ and negative if $S>p$. Hence, I(S) is an increasing function of $S$ for $S<p$ and a decreasing function of $S$ for $S>p$. From (5), we see that $I(0)=-\infty$ and $I\left(S_{0}\right)=I(0)>0$. Therefore, there exists a unique point $S_{\infty}, 0<S_{\infty}<S_{0}$, such that $I\left(S_{\infty}\right)=0$ and $I(S)>0$ for $S_{\infty}<S \leq S_{0}$. The point $\left(S_{\infty}, 0\right)$ is an equilibrium point of the first two equations of $(1)$ since both $\frac{d S}{d t}$ and $\frac{d l}{d t}$ vanish when $I=0$. 
Lemma 2.4 Let $(S(t), I(t), R(t))$ be a solution of the $S I R$ model $(1)-(2)$ in $T=\{(S, I): S \geq 0, I \geq 0, S+I \leq N\}$. Then $0<S(t) \leq S_{0}$ and

$$
S(t)=S_{0} e^{\frac{\beta(R(t)-R(0))}{\mu}} \geq S_{0} e^{-\frac{\beta N}{\mu}} \text { for all } t>0 \text {. }
$$

Proof By dividing the first and third equations of (1), we obtain that $\frac{d S}{d R}=-\frac{\beta S}{\mu}$. Thus $\int \frac{d S}{S}=\int-\frac{\beta}{\mu} d R$. By (2), we know that $\ln \left(\frac{S(t)}{S(0)}\right)=-\frac{\beta}{\mu} R(t)+\frac{\beta}{\mu} R(0)$, so that $\ln (S(t))=\ln (S(0))+\frac{\beta\left(R_{0}-R(t)\right)}{\mu}$. We conclude that $S(t)=S_{0} e^{-\frac{\beta\left(R(t)-R_{0}\right)}{\mu}}$. From Lemma 2.2, $0 \leq R(t) \leq N$ and we obtain that $S_{0} e^{-\frac{\beta N}{\mu}} \leq S_{0} e^{-\frac{\beta\left(R(t)-R_{0}\right)}{\mu}} \leq S_{0}$. Because $S_{0}>0$, we conclude that $0<S(t) \leq S_{0}$ for all $t \geq 0$.

The first conclusion we get from Lemma 2.4 is that $S$ is always a positive value, hence there always remains some susceptible who are never infected. We can also compute the number of susceptible at any time $t$ by using (6). The second conclusion is: if a small group of infectious is inserted into a group of susceptible $S_{0}$ and $S_{0}<p$, then the virus will die out rapidly. On the other hand, if $S_{0}>p$, then $I(t)$ increases as $S(t)$ decreases to $p$, where it achieves its maximum value.

Lemma 2.5 Let $(S(t), I(t), R(t))$ be a solution of the SIR model (1)-(2). Then $S(t) \rightarrow S_{\infty}$ as $t \rightarrow \infty$ and $R(t) \rightarrow R_{\infty}$ as $t \rightarrow \infty$, where $S_{\infty}$ and $R_{\infty}$ are finite numbers.

Proof From the first equation of (1) and Lemma 2.1, we have $\frac{d S(t)}{d t}=-\beta S(t) I(t) \leq 0$ so that $S(t)$ is a decreasing function and $\lim _{t \rightarrow \infty} S(t)=S_{\infty}$, where $S_{\infty}$ is a finite number. From the third equation of $(1)$, we have $\frac{d R(t)}{d t}=\mu l(t) \geq 0$ so that $R(t)$ is an increasing function. By Lemma 2.2, $\lim _{t \rightarrow \infty} R(t)=R_{\infty}$, where $R_{\infty}$ is a finite number.

Lemma 2.6 Let $(S(t), I(t), R(t))$ be a solution of the SIR model (1)-(2). Then $I(t) \rightarrow 0$ as $t \rightarrow \infty$.

Proof By (1)-(2), and knowing that from Lemma $2.5 \lim _{t \rightarrow \infty} R(t)=R_{\infty}$, so that $\lim _{t \rightarrow \infty} \mu \int_{0}^{t} I(s) d s=\frac{R_{\infty}}{\mu}$, we obtain

$$
\int \frac{d R}{d t}=\int \mu / d t \Rightarrow R(t)=\mu \int_{0}^{t} I(s) d s \Rightarrow \lim _{t \rightarrow \infty} \frac{R(t)}{\mu}=\lim _{t \rightarrow \infty} \mu \int_{0}^{t} I(s) d s .
$$

Therefore, $\lim _{t \rightarrow \infty} \int_{0}^{t} I(s) d s$ converge. Thus $\sum_{n=0}^{\infty} I(n)$ is convergent and $\lim _{t \rightarrow \infty} I(t)=0$ [33].

We have shown that $0 \leq S(t), I(t), R(t) \leq N$ over the entire time course and $S(t), R(t)$ and $I(t)$ converge to finite numbers as $t \rightarrow \infty$. These results are important in that they allow us to examine the model even further. The important point we want to address now is: how many secondary infectious appear in the population after we introduce one infective into it? If everyone is initially susceptible $\left(S_{0} \approx N\right)$, then

$$
\frac{d l}{d t}=\beta S I-\mu I=(\beta S-\mu) I \approx(\beta N-\mu) I .
$$

It means a newly introduced infected individual can be expected to infect other people at the rate $\beta N$ during the expected infectious period $\frac{1}{\mu}$. Thus, this first infective individual can be expected to infect $R_{0}=\frac{\beta N}{\mu}=\frac{N}{p}$ individuals. The number $R_{0}$ is called the basic reproduction number, which is one of the most important quantities to be considered when analyzing any epidemic model. It is a decreasing function for $R_{0}<1$, in which case the population of infectious dies out naturally. However, if $R_{0}>1$, then it is an increasing function and the virus spreads. More precisely, the virus spreads if $N>1$, respectively $S_{0}>p$, i.e., the initial number of susceptible exceeds the threshold value $p$; otherwise the virus will die out. To sum it up: if a small group of infectious is inserted into a group of susceptible $S_{0}$ and $S_{0}<p$, then the virus will die out rapidly. On the other hand, if $S_{0}>p$, then $I(t)$ increases as $S(t)$ decreases to $p$, where it achieves its maximum value, then $I(t)$ starts to decrease (when $S(t)$ falls bellow the threshold value $p)$. These conclusions are summarized in the following result.

Theorem 2.1 Let $(S(t), I(t), R(t))$ be a solution of the SIR model $(1)-(2)$ in $T=\{(S, I): S \geq 0, I \geq 0, S+I \leq N\}$. If $S_{0}<p$, then $I(t)$ decreases to 0 as $t \rightarrow \infty$. If $S_{0}>p$, then $I(t)$ increases until it attains its maximum value. After that point, and according to Lemma 2.6, I $(t)$ decreases to 0 as $t \rightarrow \infty$; also, $S(t)$ is a decreasing function and the limiting value $S_{\infty}$ is the unique root of the equation $I_{0}+S_{0}-S_{\infty}+p \ln \left(\frac{S_{\infty}}{S_{0}}\right)=0$. 


\subsection{Analysis of the SEIR model}

We also prove some fundamental properties of the SEIR system (3)-(4).

Proposition 2.2 The total population $\hat{N}$ being modelled by the SEIR model (3) is constant in time, that is, $\hat{S}(t)+\hat{E}(t)+$ $\hat{l}(t)+\hat{R}(t)=\hat{N}$ for all $t \geq 0$.

Proof Follows by adding the right-hand side of equations $(3): \hat{S}^{\prime}(t)+\hat{E}^{\prime}(t)+\hat{l}^{\prime}(t)+\hat{R}^{\prime}(t)=0$.

Lemma 2.7 Let $(S(t), E(t), I(t), R(t))$ be a solution of the SEIR model (3)-(4). Then $S(t) \geq 0, E(t) \geq 0$ and $I(t) \geq 0$, for all $t \geq 0$

Proof The proof is done by contradiction. We have that $S(t)$ is a continuous function and $S(0)=\hat{S}_{0}>0$ is the initial condition. Suppose that $S\left(t^{\prime}\right)<0$. Then, by applying the intermediate value theorem, there exist $t_{1} \in\left[0, t^{\prime}\right]$ such that $S\left(t_{1}\right)=0$. By using the first equation of (3), we obtain $S(t)=S\left(t_{1}\right) e^{f}=0$ for $t \geq t_{1}$, where $f$ is the primitive of $-\hat{\beta} /(t)$. So $S(t)=0$ for $t \geq t_{1}$, which is a contradiction. Then $S(t) \geq 0$. Similarly, we prove that $E(t) \geq 0$ and $I(t) \geq 0$.

Lemma 2.8 Let $(S(t), E(t), I(t), R(t))$ be a solution of the SEIR model (3)-(4). Then $0 \leq R(t) \leq \hat{N}, S(t) \leq \hat{N}, I(t) \leq \hat{N}$, and $E(t) \leq \hat{N}$ for all $t \geq 0$.

Proof From the fourth equation of (3), we have $\frac{d R(t)}{d t}=\hat{\mu} /(t)$. Then $\frac{d R(t)}{d t} \geq 0$ for all $t \geq 0$ and $R(t) \geq \hat{R}_{0}=0$. By Lemma 2.8, we know that $S(t) \geq 0, E(t) \geq 0$ and $I(t) \geq 0$, so $-S(t)-E(t)-I(t) \leq 0$. Therefore, $R(t)=\hat{N}-S(t)-$ $E(t)-I(t) \leq \hat{N}$, and $R(t) \leq \hat{N}$ for all $t \geq 0$. For the susceptible individuals, $S(t)=\hat{N}-E(t)-I(t)-R(t) \leq \hat{N}$ because $E(t)+I(t)+R(t) \geq 0$. Similarly, $E(t) \leq \hat{N}$ and $I(t) \leq \hat{N}$.

Lemma 2.9 Let $(S(t), E(t), I(t), R(t))$ be a solution of the SEIR model (3)-(4). Then $R(t) \rightarrow \hat{R}_{\infty}$ as $t \rightarrow \infty$, where $\hat{R}_{\infty}$ is a finite number.

Proof From the fourth equation of (3), we have $\frac{d R}{d t}=\hat{\mu} /(t) \geq 0$, so that $R(t)$ is an increasing function. By Lemma 2.8, $R(t)$ is bounded. Therefore, $\lim _{t \rightarrow \infty} R(t)=\hat{R}_{\infty}$, where $\hat{R}_{\infty}$ is a finite number.

Lemma 2.10 Let $(S(t), E(t), I(t), R(t))$ be a solution of the SEIR model (3)-(4). Then $I(t) \rightarrow 0$ as $t \rightarrow \infty$.

Proof By using (3), and knowing from Lemma 2.9 that $\lim _{t \rightarrow \infty} R(t)=\hat{R}_{\infty}$, so that $\lim _{t \rightarrow \infty} \hat{\mu} \int_{0}^{t} I(s) d s=\frac{\hat{R}_{\infty}}{\hat{\mu}}$, we obtain

$$
\int \frac{d R}{d t}=\int \hat{\mu} / d t \Rightarrow R(t)=\hat{\mu} \int_{0}^{t} I(s) d s \Rightarrow \lim _{t \rightarrow \infty} \frac{R(t)}{\hat{\mu}}=\lim _{t \rightarrow \infty} \hat{\mu} \int_{0}^{t} I(s) d s
$$

Therefore, $\lim _{t \rightarrow \infty} \int_{0}^{t} I(s) d s$ converges, so $\sum_{n=0}^{\infty} I(n)$ is convergent [33]. We conclude that $I(t) \rightarrow 0$ as $t \rightarrow \infty$.

\section{Numerical simulation of the models}

Now we investigate the numerical simulation of the SIR and SEIR models proposed for the description of the transmission of the Ebola virus in the recent outbreak occurred in West Africa $[24,25]$. In the numerical resolution of the nonlinear differential equations of both SIR and SEIR models, we use the Matlab differential equation solver ode45. This routine uses a variable step Runge-Kutta 4th/5th-order method to solve numerically the differential equations.

Rachah and Torres considered the system of equations that describe the SIR model by using the transmission rate parameter $\beta=0.2$, the recovered rate $\mu=0.1$ and the values $(S(0), I(0), R(0))=(0.95,0.05,0)$ for the initial number of susceptible, infected, and recovered individuals. These parameters of the SIR model were identified by using recent data from the World Health Organisation (WHO) [24]. Also motivated by the data of $\mathrm{WHO}$, in their numerical study of the propagation of the virus by the SEIR model, they considered the SEIR model with a transmission rate $\hat{\beta}=0.2$, an infectious rate $\hat{\gamma}=0.1887$, a recovered rate $\hat{\mu}=0.1$, and initial values $(\hat{S}(0), \hat{E}(0), \hat{l}(0), \hat{R}(0))=(0.88,0.07,0.05,0)$ for the initial number of susceptible, exposed, infected, and recovered individuals [25]. The numerical simulations of the SIR and SEIR models were carried out in the previous works of Rachah and Torres [24,25]. Here, by using the above mentioned parameters and initialization values, we investigate the possibility to obtain curves of infectious that converge at the same time and have the same maximum numbers of infectious individuals by the two models.

After an extensive set of numerical tests of the models with several values of $\hat{\gamma}$, we concluded that convergence between the results of the two models is possible by increasing the infectious rate. In fact, when $\hat{\gamma}$ increases, the individuals enter the / class as soon as the individuals enter the class $E$. In that case, the SIR model can be viewed as a special case of the SEIR model. In the 


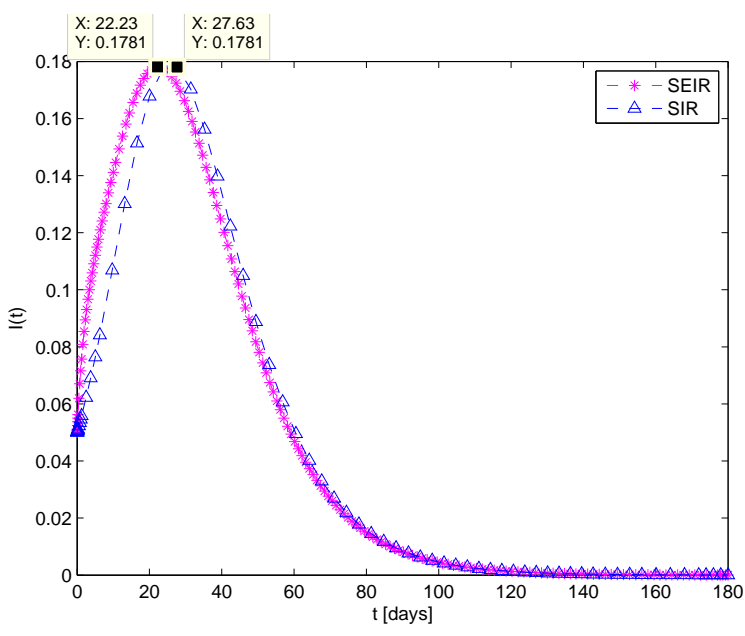

(a) Infectious individuals

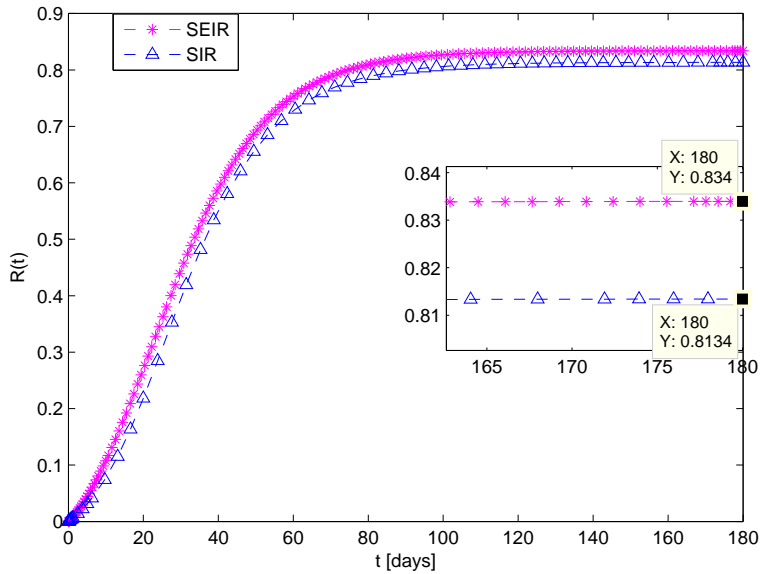

(b) Recovered individuals

Figure 2. Comparison between the numerical solution of the SIR and SEIR models in the case of $\hat{\gamma}=2.47 \times 0.1887$.

analysis and comparison between the numerical resolution of the SIR and SEIR models, we used the real parameters identified from the real data of the WHO given by $\hat{\beta}=\beta=0.2, \hat{\mu}=\mu=0.1$, the initial values $(\hat{S}(0), \hat{E}(0), \hat{l}(0), \hat{R}(0))=(0.88,0.07,0.05,0)$, and several coefficients values of $\hat{\gamma}$. Our comparison between the numerical results of the SIR and SEIR models is shown in Figure 2. The numerical study of the models shows that they have the same maximum number and a convergence of the curve of infectious at the same time for the value of infectious rate $\hat{\gamma}=2.47 \times 0.1887$. The meaning of this value is that when $\hat{\gamma}$ increases, the number of exposed individuals decreases. Then we obtain a convergence between the curves of infectious of the two models.

For the SEIR model, we chose $\hat{\gamma}=2.47 \times 0.1887$ with a small value of $E(0), \hat{\beta}=\beta, \hat{\mu}=\mu$ and $(\hat{I}(0), \hat{R}(0))=(I(0), R(0))$, in order to discuss the difference between the models in case when they have the same maximum number of infectious with similar curves of infectious. Figure 2 shows the curve of infectious individuals of the SIR model, which is identical to the SEIR curve of infectious individuals. The same figure shows that the maximum number of infectious individuals is the same for the two models and the curves converge at the same time, and presents the number of recovered individuals for the two models and for the same maximum number of infectious. By using the SEIR model, the recovered number of individuals is more important than the number of recovered individuals of the SIR model. The numerical results show that even when one model converge to the other one (with respect to the infectious number), by increasing $\hat{\gamma}$ the SEIR model describes better the propagation of the virus, which is closer to the reality of Ebola virus, characterized by a period of incubation described by the exposed group. Even when the number of exposed is very small, it provides a more detailed description of the Ebola virus.

\section{Optimal control of the virus by using SIR and SEIR models}

We now address the question of how to optimally control the propagation of the spread of Ebola in a population by using the SIR and SEIR models. Let us start by the optimal control strategy based on the SIR model, as studied by Rachah and Torres in [24], which is given by the following system of nonlinear differential equations:

$$
\left\{\begin{array}{l}
\frac{d S(t)}{d t}=-\beta S(t) I(t)-u(t) S(t), \\
\frac{d I(t)}{d t}=\beta S(t) I(t)-\mu I(t), \\
\frac{d R(t)}{d t}=\mu I(t)+u(t) S(t) .
\end{array}\right.
$$

Note that if $u(t) \equiv 0$, then (7) reduces to (1). Although there are currently no licensed Ebola vaccines, two potential candidates are undergoing evaluation. The goal of the strategy is to reduce the infected individuals and the cost of vaccination. Precisely, the optimal control problem consists of minimizing the objective functional

$$
J(u)=\int_{0}^{t_{\text {end }}}\left[I(t)+\frac{\nu}{2} u^{2}(t)\right] d t
$$

where $u(t)$ is the control variable, which represents the vaccination rate at time $t$, and the parameters $\nu$ and $t_{\text {end }}$ denote, respectively, the weight on cost and the duration of the vaccination program. 
Let us now study the same cost functional of the previous optimal control problem, by introducing into the model (3) a control $\tilde{u}(t)$, representing the vaccination rate at time $t$. The control $\tilde{u}(t)$ is the fraction of susceptible individuals being vaccinated per unit of time. Then, the mathematical model with control is given by the following system of nonlinear differential equations:

$$
\left\{\begin{array}{l}
\frac{d \tilde{S}(t)}{d t}=-\tilde{\beta} \tilde{S}(t) \tilde{I}(t)-\tilde{u}(t) \tilde{S}(t) \\
\frac{d \tilde{E}(t)}{d t}=\tilde{\beta} \tilde{S}(t) \tilde{I}(t)-\tilde{\gamma} \tilde{E}(t) \\
\frac{d \tilde{I}(t)}{d t}=\tilde{\gamma} \tilde{E}(t)-\tilde{\mu} \tilde{I}(t) \\
\frac{d \tilde{R}(t)}{d t}=\tilde{\mu} \tilde{I}(t)+\tilde{u}(t) \tilde{S}(t) .
\end{array}\right.
$$

The goal of our strategy is to reduce the infected individuals and the cost of vaccination. Precisely, the optimal control problem consists of minimizing the objective functional

$$
J(\tilde{u})=\int_{0}^{t_{\text {end }}}\left[\tilde{I}(t)+\frac{\tilde{\tau}}{2} \tilde{u}^{2}(t)\right] d t
$$

where $\tilde{u}(t)$ is the control variable, which represents the vaccination rate at time $t$, and the parameters $\tilde{\tau}$ and $t_{\text {end }}$ denote, respectively, the weight on cost and the duration of the vaccination program.

Before comparing between the results of control of the two models, we present in Figure 3 the numerical simulation of the SIR model without control compared with the study of control of the virus described by the system (7) and the cost functional (8). Figure 3 shows the effect of the optimal control strategy in reducing the number of infectious and the period of infection, and in increasing the number of recovered.

Figure 4 shows the numerical simulation of the SEIR model without control compared with the study of control of the virus described by the system (9) and the cost functional (10). The curves of the compartments in Figure 4 show the effect of the optimal control strategy in reducing the number of exposed and infectious individuals and the period of infection, and in increasing the number of recovered individuals.

Let us now discuss the comparison between the results of the control strategy of the SIR and SEIR models. Figure 5a shows that the number of susceptible decreases faster in case of control of the virus by using the SEIR model than the case of control by the SIR model. Figure 5b presents the number of infectious individuals, which is characterized by a peak in the same time (4 days) for the two models with a number of infected given by $6.7 \%$ in case of control by the SEIR model and $5.6 \%$ in the case of control by the SIR model. The difference in the number of infectious between the two models is explained by the fact that the exposed individuals certainly moved to the infectious class since the Ebola virus is characterized by a short latent period, then it spreads very quickly. The effect of the control strategy is shown in the period of infection, which is given by 66 days in case of control by the SEIR model, which is less than the infectious period (78 days) in case of control by using the SIR model. The number of recovered individuals is shown in Figure 5c, where it reaches $99 \%$ at the end of the campaign in case of control by the SEIR model versus $88 \%$ at the end of the campaign in case of control with the SIR model. Figure $5 d$ presents the control variable. One can see that the control is more important in the SEIR model than in the SIR case. More precisely, Figure $5 d$ shows that the optimal control function of the SEIR model starts at the upper bound 0.9, while the optimal control of the SIR model starts at 0.2. This fact is explained by the severity of the spread of the virus, which requires an immediate implementation against the virus, and the superiority of the SEIR system in modelling Ebola: the exposed compartment of the SEIR model shows the severity of the virus, where individuals can be infected in the beginning, without symptoms, and then transmission blows up during the latent period. Thus, the rate of vaccination is more crucial in case of the control strategy based on the SEIR model, which is closer to the reality of the propagation of the Ebola virus.

\section{Conclusion}

We presented a comparison study between the SIR and SEIR models used in the description of the propagation of the Ebola virus. The models were compared by using their numerical simulation and also by studying optimal control strategies for the control of the virus. Our investigations are based on the parameters previously identified by Rachah and Torres in their study of the Ebola virus [24, 25].

\section{Acknowledgement}

This research was supported by the Institut de Mathématiques de Toulouse (Rachah); and by the Portuguese Foundation for Science and Technology (FCT), within R\&D unit CIDMA, project UID/MAT/04106/2013 (Torres). The authors would like to thank two reviewers for their comments and suggestions. 


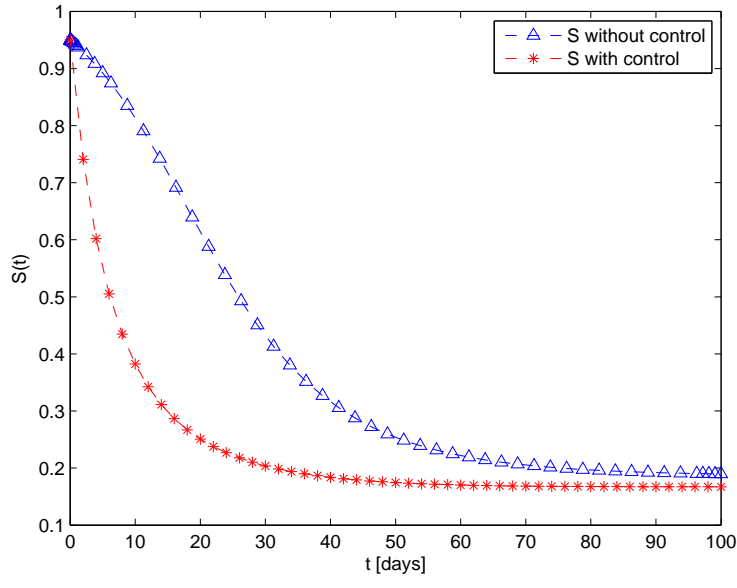

(a) Susceptible with and without control

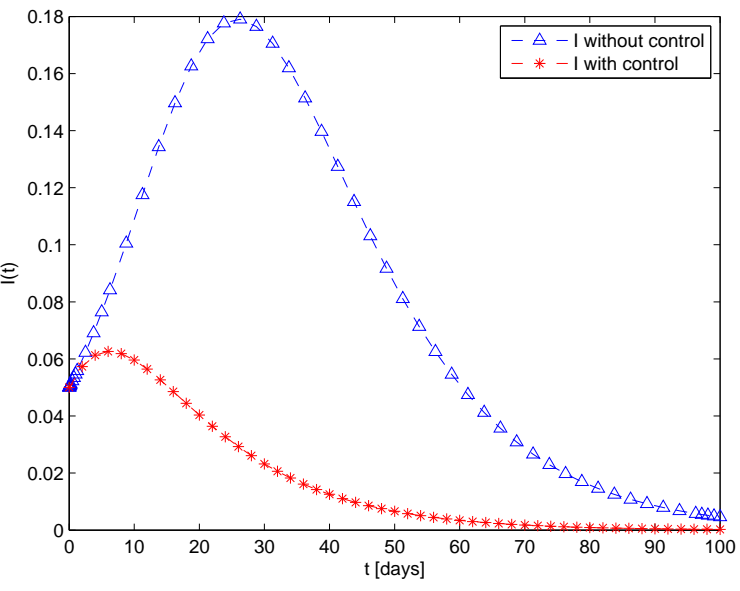

(b) Infectious with and without control

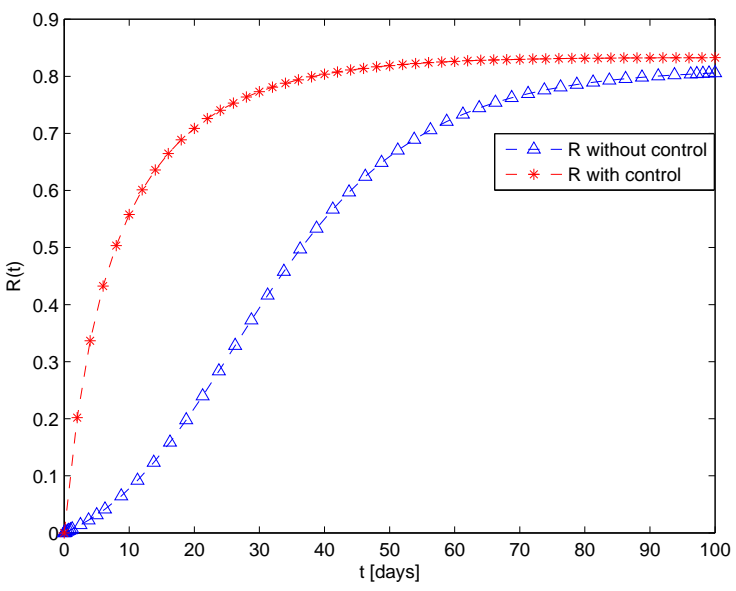

(c) Recovered with and without control

Figure 3. Simulation of Ebola virus and numerical resolution of a strategy control of the Ebola virus by using the SIR control model described by system (7) and the cost functional (8)

\section{References}

1. Barry M, Traoré F A, Sako F B, Kpamy D O, Bah E I, Poncin M, Keita S, Cisse M, Touré A. 2014. Ebola outbreak in Conakry, Guinea: Epidemiological, clinical, and outcome features. Médecine et Maladies Infectieuses 44, no. 11-12, 491-494.

2. Lewnard J A, Ndeffo Mbah M L, Alfaro-Murillo J A, Altice F L, Bawo L, Nyenswah T G, Galvani A P. 2014. Dynamics and control of Ebola virus transmission in Montserrado, Liberia: A mathematical modelling analysis. The Lancet Infectious Diseases 14, no. 12, $1189-1195$

3. Legrand J, Grais R F, Boelle P Y, Valleron A J, Flahault A. 2007. Understanding the dynamics of Ebola epidemics. Epidemiol. Infect. 135 , no. 4, 610-621.

4. WHO, Report of an International Study Team. 1978. Ebola haemorrhagic fever in Sudan 1976. Bull. World Health Organ. 56, no. 2, 247-270.

5. Peters C J, LeDuc J W. 1999. An introduction to Ebola: The virus and the disease. J. Infect Dis. 179, Suppl. 1, ix-xvi.

6. Smith C T, Hilary Babcock M. D. 2010. Ebola and Marburg virus (deadly diseases and epidemics). Chelsea House Publisher.

7. Alton J. 2014. The Ebola survival handbook. Skyhorse Publishing New York.

8. Borio L et al. [Working Group on Civilian Biodefense; Corporate Author]. 2002. Hemorrhagic fever viruses as biological weapons: medical and public health management. Journal of the American Medical Association 287, no. 18, 2391-2405.

9. Dowell S F, Mukunu R, Ksiazek T G, Khan A S, Rollin P E, Peters C J. 1999. Transmission of Ebola hemorrhagic fever: A study of risk factors in family members, Kikwit, Democratic Republic of the Congo, 1995. Commission de Lutte contre les Epidémies à Kikwit. J. Infect. Dis. 179, Suppl. 1, S87-S91.

10. Chapnick E K. 2015. Ebola Myths \& Facts. Wiley \& Sons Publisher.

11. Smith C T. 2006. Ebola. Deadly Diseases and Epidemics. Chelsea House Publisher. 


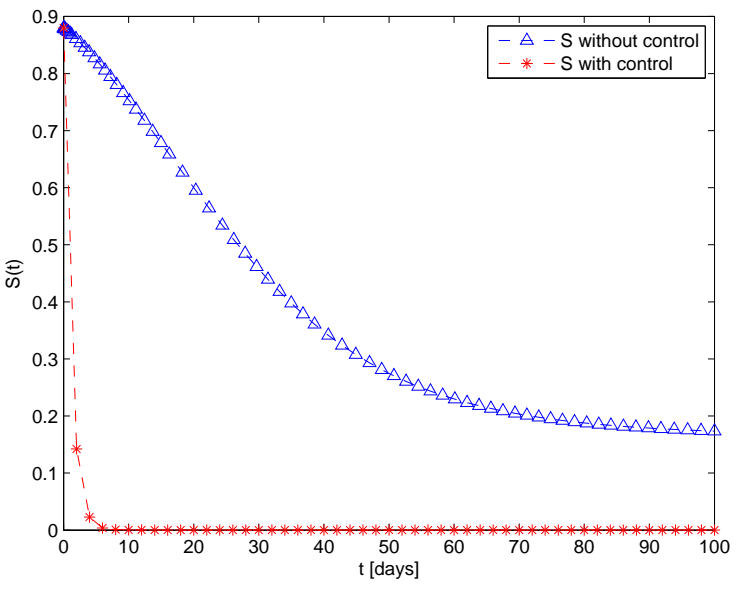

(a) Susceptible with and without control

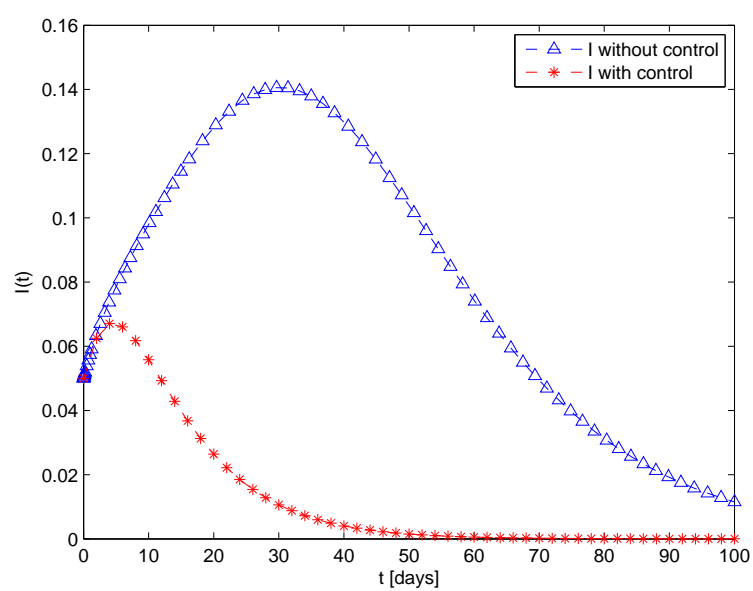

(c) Infectious with and without control

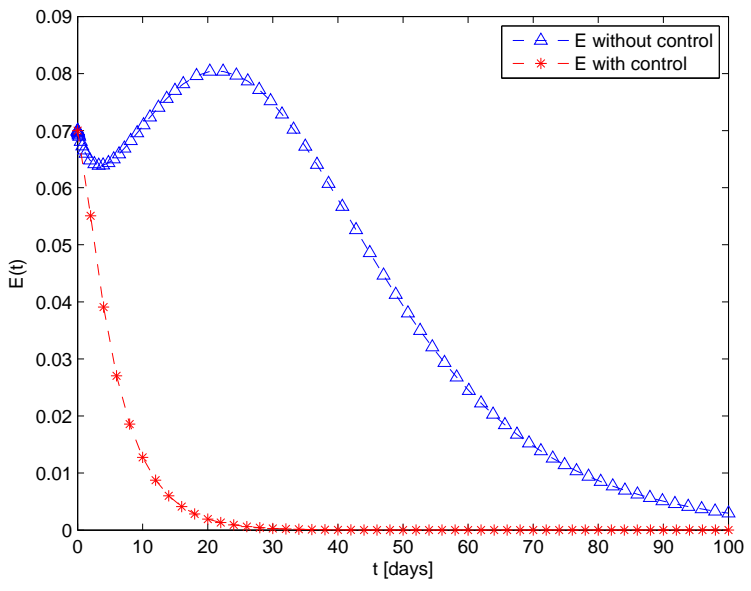

(b) Exposed with and without control

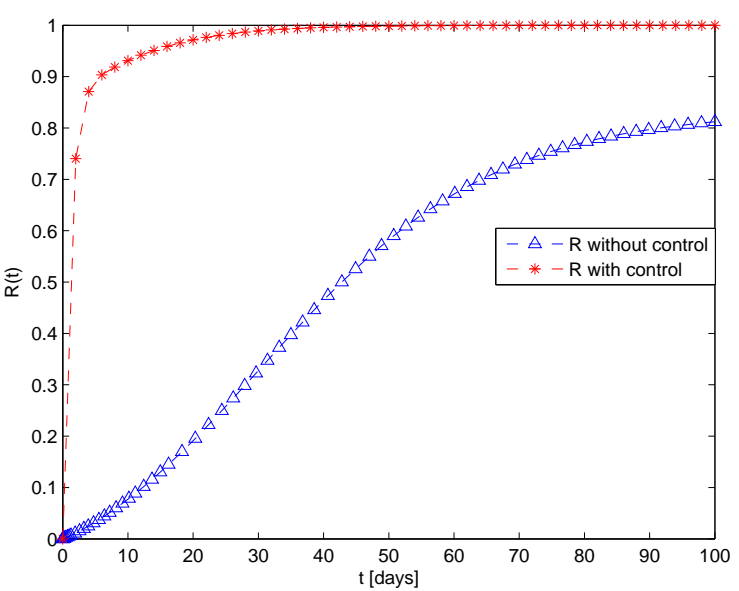

(d) Recovered with and without control

Figure 4. Simulation of Ebola virus and numerical resolution of a strategy control of the Ebola virus by using the SEIR control model described by system (9) and the cost functional (10).

12. Report of an International Commission. 1978. Ebola haemorrhagic fever in Zaire, 1976. Bull. World Health Organ. 56, no. 2, 271-293.

13. Uganda Ministry of Health. 2002. An outbreak of Ebola in Uganda. Trop. Med. Int. Health. 7, no. 12, 1068-1075.

14. Diekmann O, Heesterbeek H, Britton T. 2013. Mathematical tools for understanding infectious disease dynamics. Princeton Series: In theoretical and Computational Biolgy, ISBN 978-0-691-15539-5.

15. Rodrigues H S, Monteiro M T T, Torres D F M. 2014. Vaccination models and optimal control strategies to dengue. Math. Biosci. 247, no. 1, 1-12. arXiv: 1310.4387

16. Rodrigues H S, Monteiro M T T, Torres D F M. 2010. Dynamics of dengue epidemics when using optimal control. Math. Comput. Modelling 52, no. 9-10, 1667-1673. arXiv:1006.4392

17. Longini Jr I M, Ackerman E. 1978. An optimization model for influenza A epidemics. Mathematical Biosciences 38, no. 1-2, 141-157.

18. Kretzschmar M. 2004. Ring vaccination and smallpox control. Emerging Infectious Diseases, 10, no. 5, 832-841.

19. Kermack W, McKendrick A. 1991. Contributions to the mathematical theory of epidemics-I. 1927. Bulletin of Mathematical Biology 53, no. 1-2, 33-55

20. Kermack W, McKendrick A. 1991. Contributions to the mathematical theory of epidemics-II. The problem of endemicity. 1932. Bulletin of Mathematical Biology 53, no. 1-2, 57-87.

21. Kermack W, McKendrick A. 1991. Contributions to the mathematical theory of epidemics-III. Further studies of the problem of endemicity. 1933. Bulletin of Mathematical Biology 53, no. 1-2, 89-118.

22. Hethcote W H. 2000. The Mathematics of Infectious Diseases. SIAM Review. Vol. 42, No. 4, 599-653.

23. Brauer F, Driessche P V D, Wu J. 2008. Mathematical Epidemiology. Lectures Notes in Mathematics 1945, Mathematical Biosciences Subseries.

24. Rachah A, Torres D F M. 2015. Mathematical modelling, simulation and optimal control of the 2014 Ebola outbreak in West Africa. Discrete Dyn. Nat. Soc., Art. ID 842792, 9 pp. arXiv:1503.07396 


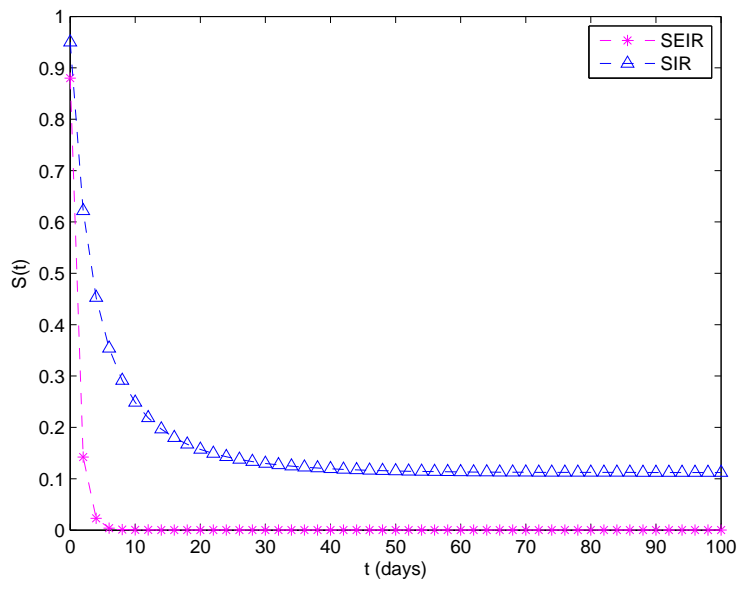

(a) Susceptible

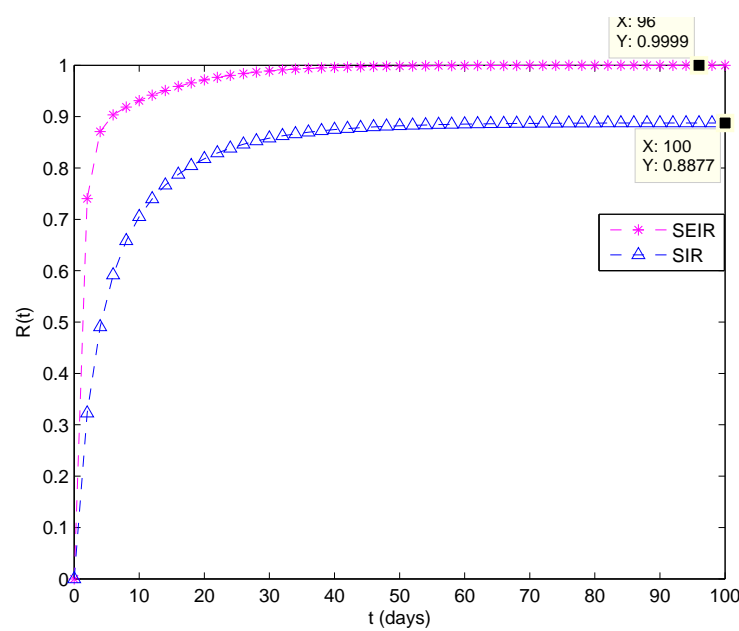

(c) Recovered

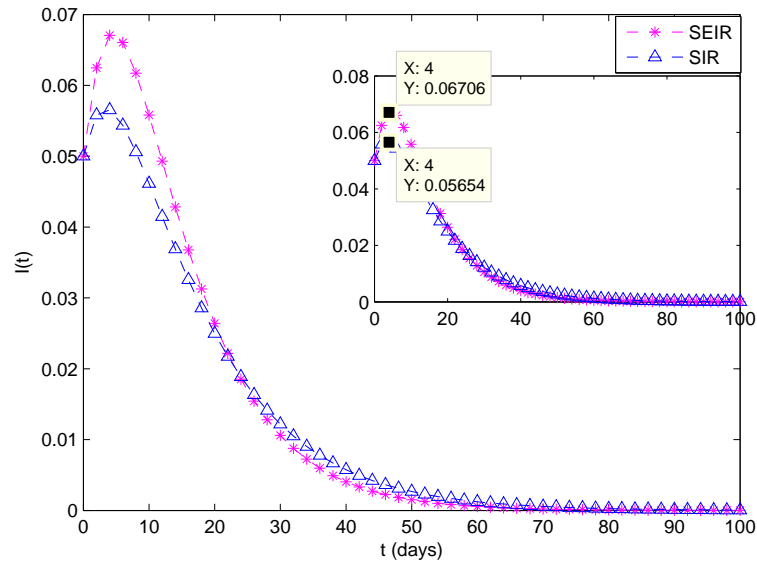

(b) Infectious

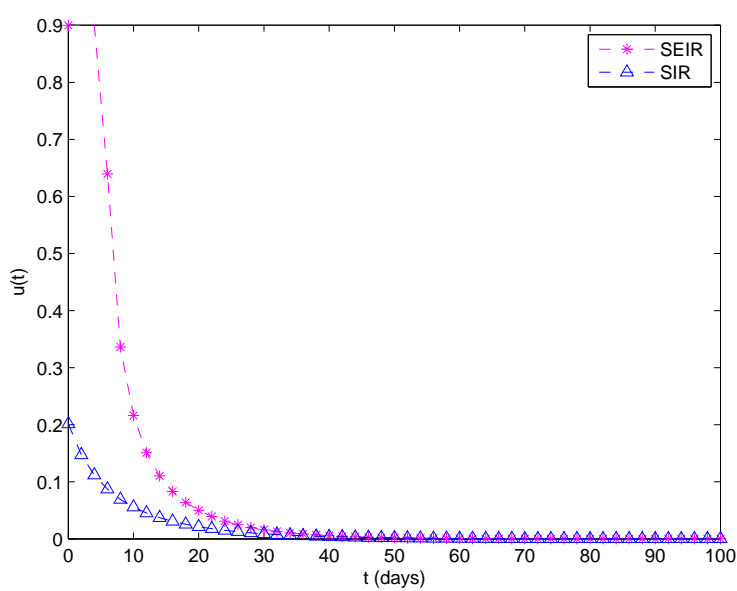

(d) Optimal control

Figure 5. Comparison of control of Ebola virus by the SIR model (described by system (7) and the cost functional (8)) versus control by the SEIR model (described by system (9) and the cost functional (10)).

25. Rachah A, Torres D F M. 2015. Modelling and numerical simulation of the recent outbreak of Ebola, 2nd International Conference on Numerical and Symbolic Computation: Developments and Applications (SYMCOMP 2015), pp. 179-190. ISBN 978-989-96264-7-8.

26. Gaff H, Schaefer E. 2009. Optimal control applied to vaccination and treatment strategies for various epidemiological models. Math Biosci Eng., 6, no. 3, 469-492.

27. Banks H T, Castillo-Chavez C. 1987. Bioterrorism. SIAM Frontiers In Applied Mathematics.

28. James-Berry C M. 2013. Vaccine Control of Avian Influenza H5N1 in Poultry: Need for a Positive Marker. Journal Vaccines \& Vaccin., 4:168. doi: 10.4172/2157-7560.1000168

29. Gao S, Teng Z, Nieto Juan J, Torres A. 2007. Analysis of an SIR Epidemic Model with Pulse Vaccination and Distributed Time Delay. Journal of Biomedicine and Biotechnology, Article ID 64870

30. Jung E, Iwami S, Takeuchi Y, Jo T C. 2009. Optimal control strategy for prevention of avian influenza pandemic. Journal of Theoretical Biology, 260, 220-229.

31. Tchuenche J M, Khamis S A, Agusto F B, Mpeshe S C. 2011. Optimal control and sensitivity analysis of an influenza model with treatment and vaccination. Acta Biotheoretica, 59, no. 1, 1-28.

32. Area I, Batarfi H, Losada J, Nieto J J, Shammakh W, Torres A. 2015 On a fractional order Ebola epidemic model. Adv. Difference Equ. 2015, 278.

33. Bloch E D. 2011. The Real Numbers and Real Analysis. Springer, New York. Dordrecht, Heidelberg, London. 